Biotheranostics, Exelixis, Bristol-Myers-Squibb, Janssen, Amgen, and Eisai; receives personal fees from NCCN (National Comprehensive Cancer Network); and receives institutional research funding and support from Boehringer-Ingelheim, Bayer, Onyx-Amgen, and Merck. TKC receives honoraria for Alligent, AstraZeneca, Bayer, Bristol-Myers Squibb, Cerulean Pharma, Eisai, Exelixis, Foundation Medicine, GlaxoSmithKline, Merck, Novartis, Peloton, Pfizer, Prometheus, and Roche/Genentech; institutional research funding and support from Pfizer, Exelixis, Bristol-Myers Squibb, Novartis, Peloton, AstraZeneca, Agensys, and TRACON. A-KAL declares no competing interests.

1 Rosenberg JE, Hoffman-Censits J, Powles T, et al. Atezolizumab in patients with locally advanced and metastatic urothelial carcinoma who have progressed following treatment with platinum-based chemotherapy: a single-arm, multicentre, phase 2 trial. Lancet 2016; 387: 1909-20.

2 Sharma P, Retz M, Siefker-Radtke A, et al. Nivolumab in metastatic urothelial carcinoma after platinum therapy (CheckMate 275): a multicentre, single-arm, phase 2 trial. Lancet Oncol 2017; 18: 312-22.

3 Bellmunt J, de Wit R, Vaughn DJ, et al. Pembrolizumab as second-line therapy for advanced urothelial carcinoma. N Engl J Med 2017; 376: 1015-26.

4 Powles T, O'Donnell PH, Massard C, et al. Efficacy and safety of durvalumab in locally advanced or metastatic urothelial carcinoma: updated results from a phase 1/2 open-label study. JAMA Oncol 2017; 3: e172411.
5 Patel MR, Ellerton J, Infante JR, et al. Avelumab in metastatic urothelial carcinoma after platinum failure (JAVELIN Solid Tumor): pooled results from two expansion cohorts of an open-label, phase 1 trial. Lancet Oncol 2017; published online Dec 4. http://dx.doi.org/10.1016/S14702045(17)30900-2.

6 Pond GR, Sonpavde G, Rosenberg JE, et al. Nomogram to assess benefit of new over historical agents as salvage therapy for metastatic urothelial carcinoma ( $\mathrm{mUC}$ ) in non-randomized trials: effect of atezolizumab on 12-month survival. J Clin Oncol 2017; 35: 346.

7 Boyerinas B, Jochems C, Fantini M, et al. Antibody-dependent cellular cytotoxicity activity of a novel anti-PD-L1 antibody avelumab (MSB0010718C) on human tumor cells. Cancer Immunol Res 2015; 3: 1148-57.

8 Martini DJ, Lalani AA, Bosse D, et al. Response to single agent PD-1 inhibitor after progression on previous PD-1/PD-L1 inhibitors: a case series. J Immunother Cancer 2017; 5: 66.

9 Petrylak DP, de Wit R, Chi KN, et al. Ramucirumab plus docetaxel versus placebo plus docetaxel in patients with locally advanced or metastatic urothelial carcinoma after platinum-based therapy (RANGE): a randomised, double-blind, phase 3 trial. Lancet 2017; 390: 2266-77.

10 Powles T. IMvigor211: a phase III randomized study examining atezolizumab versus chemotherapy for platinum-treated advanced urothelial carcinoma. EACR-AACR-SIC Special Conference: Florence, Italy; June 24-27, 2017. Abstract 606.

\title{
Venetoclax: a chance for patients with chronic lymphocytic leukaemia previously treated with ibrutinib
}

In recent years, biological and clinical research has identified several targeted agents that are changing the management of patients with chronic lymphocytic leukaemia. In clinical trials, ${ }^{1}$ the B-cell receptor inhibitor ibrutinib has led to durable responses and longer survival (overall and progression-free) than chemotherapy in patients with this disease. Ibrutinib is now largely used in clinical practice in patients with chromosome 17p deletions or TP53 mutations and relapsed or refractory chronic lymphocytic leukaemia. More recently, the drug has also been approved in the USA and European Union as a front-line therapy for chronic lymphocytic leukaemia. ${ }^{1}$ However, many patients discontinue ibrutinib because of adverse events such as atrial fibrillation, infections, and cytopenias. Another common reason for treatment discontinuation is disease progression, which is observed most frequently in patients with TP53 mutations or complex karyotypes. ${ }^{2,3}$ Mutations conferring resistance to ibrutinib are also commonly found in patients with high-risk genetic landscapes or who have disease progression during ibrutinib treatment. ${ }^{4,5}$ Outcomes after ibrutinib discontinuation are poor, ${ }^{2,3}$ and few treatment options are available for such patients. Therefore, treatment options for patients with relapsed or refractory disease previously treated with ibrutinib are a primary unmet medical need in the management of chronic lymphocytic leukaemia, especially given the increasing use of this drug in clinical practice.

Venetoclax is a first-in-class, oral, selective inhibitor of $\mathrm{BCL}-2$ (apoptosis regulator $\mathrm{BCl}-2$ ), which regulates cell apoptosis. Inhibition of $\mathrm{BCL}-2$ in chronic lymphocytic leukaemia cells leads to a high pro-apoptotic effect that is independent of TP53. Deep responses with no evidence of minimal residual disease have been described in patients with relapsed or refractory disease given venetoclax monotherapy, including those with TP53 mutations. ${ }^{6,7}$ Combined with rituximab, venetoclax led to a high proportion of patients being negative for minimal residual disease in a phase $1 \mathrm{~b}$ study. ${ }^{8}$ Given the activity of venetoclax in patients with relapsed or refractory chronic lymphocytic leukaemia, including in those who have progressed on ibrutinib, ${ }^{9}$ Jeffrey Jones and colleagues ${ }^{10}$ did a multicentre, open-label, phase 2 trial to assess the activity and safety of venetoclax in patients with chronic lymphocytic leukaemia previously treated with ibrutinib; the results of an interim analysis are reported in The Lancet Oncology. Of the 91 patients included in the activity and safety analyses, 50 (55\%) had discontinued ibrutinib because of disease progression, 30 (33\%) because of adverse events, and 11 (12\%)

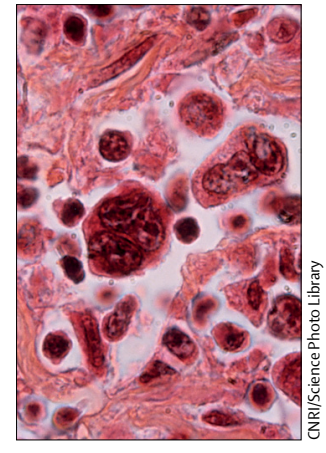

Published Online December 12, 2017 http://dx.doi.org/10.1016/ S1470-2045(17)30910-5 See Articles page 65 
because maximal clinical benefit was achieved $(n=6)$, the defined course of treatment was completed $(n=3)$ or unspecified reasons $(n=2) .{ }^{10}$

The study included patients with clinical and biological characteristics indicative of a poor prognosis and who had been heavily pre-treated, with ibrutinib being the last therapy they received before enrolment. 50 (75\%) of 67 patients were negative for mutations in immunoglobulin heavy-chain variable region genes, $42(47 \%)$ of 90 patients had a deletion in chromosome 17 [del(17)(p13.1)], and 29 (33\%) of 87 patients had a TP53 mutation. Additionally, BTK or PLCG2 mutations were found in 17 (81\%) of 21 tested patients.

59 (65\%, 95\% Cl 53-74) of the 91 patients in the study had an overall response as per investigator's assessment. Deep responses with no evidence of cytometric minimal residual disease were detected in the peripheral blood of 24 (42\%) of 57 patients who showed a response and underwent this assessment for minimal residual disease. Responses were observed independently of the reason for stopping ibrutinib and were not affected by the presence of a TP53 mutation. Notably, responses were also recorded in patients who progressed on ibrutinib because of the emergence of mutations; 12 (71\%) of 17 patients with known ibrutinib-resistance mutations had an overall response. Furthermore, a decrease in the allelic frequency of Cys481Ser BTK mutations during up to 72 weeks of venetoclax treatment was observed in eight patients with serial data available.

12-month progression-free survival was 75\% (95\% Cl 64-83) and 12-month overall survival was 91\% (83-95). These outcomes are similar to those previously reported for venetoclax in patients with relapsed or refractory disease who had previously only been treated with chemoimmunotherapy. ${ }^{4}$ Additionally, the safety profile of venetoclax was consistent with previous reports of single-drug venetoclax in the same population. ${ }^{4-6}$

The results of this trial show the capacity of venetoclax, through $\mathrm{BCL}-2$ inhibition, to overcome not only the negative effect of a TP53 mutation, but also ibrutinib resistance due to the emergence of mutations. This new information is important for optimal management of patients with chronic lymphocytic leukaemia progressing on ibrutinib. Taken together, the results of this trial are reassuring and have relevant clinical implications, especially now that ibrutinib has been approved as a first-line treatment for patients with chronic lymphocytic leukaemia. As ongoing randomised trials investigate front-line combination regimens involving venetoclax, the next question to be addressed will be how to treat patients who progress after initial therapy with this drug.

\section{*Francesca R Mauro, Robin Foà}

Department of Cellular Biotechnologies and Haematology, Sapienza University, Rome, Italy

mauro@bce.uniroma1.it

FRM was a member of an advisory board of Abbvie during the conduct of this work. RF declares no competing interests.

1 Jain N, O'Brien S. Targeted therapies for CLL: practical issues with the changing treatment paradigm. Blood Rev 2016; 30: 233-44.

2 Jain $\mathrm{P}$, Keating $\mathrm{M}$, Wierda W, et al. Outcomes of patients with chronic lymphocytic leukemia after discontinuing ibrutinib. Blood 2015: 125: 2062-67.

3 Mato AR, Nabhan C, Barr PM, et al. Outcomes of CLL patients treated with sequential kinase inhibitor therapy: a real world experience. Blood 2016; 128: 2199-205

4 Woyach JA, Ruppert AS, Guinn D, et al. BTKC481S-mediated resistance to ibrutinib in chronic lymphocytic leukemia. J Clin Oncol 2017; 35: 1437-43.

5 Jones D, Woyach JA, Zhao W, et al. PLCG2 C2 domain mutations co-occur with BTK and PLCG2 resistance mutations in chronic lymphocytic leukemia undergoing ibrutinib treatment. Leukemia 2017; 31: 1645-47.

6 Roberts AW, Davids MS, Pagel JM, et al. Targeting BCL2 with venetoclax in relapsed chronic lymphocytic leukemia. N EngJ Med 2016; 374: 311-22.

7 Stilgenbauer S, Eichhorst B, Schetelig J, et al. Venetoclax in relapsed or refractory chronic lymphocytic leukemia with $17 p$ deletion: a multicenter, open-label, phase 2 study. Lancet Oncol 2016; 7: 768-78.

8 Seymour JF, Ma S, Brander DM, et al. Venetoclax plus rituximab in relapsed or refractory chronic lymphocytic leukaemia: a phase $1 \mathrm{~b}$ study. Lancet Oncol 2017; 18: 230-40.

9 Mato AR, Hill BT, Lamanna N, et al. Optimal sequencing of ibrutinib, idelalisib, and venetoclax in chronic lymphocytic leukemia: results from a multicenter study of 683 patients. Ann Oncol 2017; 28: 1050-56.

10 Jones JA, Mato AT, Wierda WG, et al. Venetoclax for chronic lymphocytic leukaemia progressing after ibrutinib: an interim analysis of a multicentre, open-label, phase 2 trial. Lancet Oncol 2017; published online Dec 12. http://dx.doi.org/10.1016/S1470-2045(17)30909-9.

\section{(D) Bipolar androgen therapy: an intriguing paradox}

Published Online December 13, 2017 http://dx.doi.org/10.1016/ S1470-2045(17)30907-5

See Articles page 76
In The Lancet Oncology, Benjamin A Teply and colleagues ${ }^{1}$ report a phase 2 trial in men with metastatic castrationresistant prostate cancer with progression on an androgen receptor pathway inhibitor, enzalutamide, who were treated with bipolar androgen therapy (BAT). It is a novel and intriguing concept.

Castration-resistant prostate cancer occurs through numerous mechanisms, including androgen receptor 